Doctrina

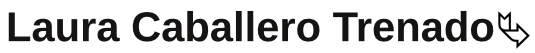

\section{Redes sociales, consumidores y propiedad intelectual. Comentario jurisprudencial a la Sentencia 14/07300, del Tribunal de Gran Instancia de París, de 7 de agosto de 2018}

Social networks, consumers and intellectual property. Case-law commentary on Judgment 14/07300, of the Court of Great Instance of Paris, of August 7, 2018

Redes sociais, consumidores e propriedade intelectual. Comentário de jurisprudência sobre o julgamento 14/07300, do Tribunal de Grande Instância de Paris, de 7 de agosto de 2018

Resumen: ¿Tienen entidad de consumidores los usuarios de redes sociales? Si la respuesta a esta pregunta es afirmativa, se activa inmediatamente un catálogo de derechos como, por ejemplo, la legitimación para accionar un procedimiento judicial. La legislación europea en materia de derechos de consumidores está armonizada y goza de una naturaleza muy tuitiva. Hasta ahora, en la interacción con las redes sociales, el usuario se veía impelido a contratar un "cheque en blanco" que suponía la cesión de buena parte de sus derechos -como, por ejemplo, los derechos de autor que pudieran corresponderle- al cesionario, el propietario de la red social. Pero la reciente Decisión del Tribunal de Gran Instancia de París ha revertido la posición jurídica de un grupo de consumidores franceses, que interpusieron una demanda colectiva contra la red social Twitter, al darles la razón, tras calificar de "abusivas" la mayoría de las cláusulas y condenar a la red social a pagar a la Asociación de consumidores francesa $30.000 \mathrm{eu}$ ros. Este trabajo analiza esta importante Resolución judicial, al objeto de trazar las implicaciones más relevantes que impactan en el derecho de los consumidores y usuarios del ámbito normativo civil, en general, y en materia de propiedad intelectual -une création du droit- (Bergé, 2015), en particular.

$\rightarrow$ Universidad Internacional de La Rioja.

0000-0001-9749-2395

laura.caballero@unir.net 
Palabras clave: redes sociales, consumidores, propiedad intelectual, cláusulas abusivas, derechos de autor.

Abstract: Do users of social networks have a consumer entity? If the answer to this question is affirmative, a catalog of rights is immediately activated, such as, for example, the legitimacy to initiate a judicial proceeding. European legislation on consumer rights is harmonized and has a very protective nature. Until now, in the interaction with social networks, the user was compelled to contract a "blank check" that involved the transfer of a large part of their rights, such as, for example, the copyright that might correspond to the transferee. The owner of the social network. But, the recent decision of the Court of Large Instance of Paris has reversed the legal position of a group of French consumers, who filed a class action against the social network Twitter, to give them the reason, after describing as "abusive" most clauses and condemn the social network to pay the French Consumer Association 30,000 euros. This paper analyzes this important judicial resolution, in order to draw the most relevant implications that impact on the right of consumers and users of the civil normative sector, in general, and in intellectual property-une création du droit- (Bergé, 2015), in particular.

Keywords: Social Networks, Consumers, Intellectual Property, Unfairterms, Copyright.

Resumo: Os usuários de redes sociais têm uma entidade de consumidor? Se a resposta a esta pergunta for afirmativa, um catálogo de direitos é imediatamente ativado, como, por exemplo, a legitimidade para iniciar um processo judicial. A legislação europeia em matéria de direitos dos consumidores é harmonizada e tem um carácter muito moderado. Até o momento, na interação com as redes sociais, o usuário era obrigado a contratar um "cheque em branco" que envolvia a transferência de grande parte de seus direitos, como, por exemplo, os direitos autorais que poderiam corresponder ao cessionário. , o dono da rede social. Mas a recente decisão do Tribunal de Grande Instância de Paris reverteu a posição legal de um grupo de consumidores franceses, que entrou com uma ação coletiva contra a rede social Twitter, para lhes dar a razão, depois de descrever como cláusulas "abusivas" e condenar a rede social a pagar à Associação Francesa de Consumidores 30.000 euros. Este artigo analisa essa importante resolução judicial, a fim de traçar as implicações mais relevantes que impactam o direito dos consumidores e usuários do campo normativo civil, em geral, e em matéria de propriedade intelectual-une création du droit- (Bergé: 2015, 5), em particular.

Palabras-chave: Redes sociais, consumidores, propriedade intelectual, cláusulas abusivas, direitos autorais.

Recibido: 20181015

Aceptado: 20190310 


\section{Introducción}

En el mundo de la información digital, si no estás pagando por un producto, eres el producto. Si las empresas continúan incrementando la cantidad de datos que almacenan sobre los individuos, los datos en sí se convierten en un producto a la venta y, si los individuos no comienzan a protegerse a sí mismos y la información digital que divulgan, corren el riesgo de que sus datos personales se agrupen y se vendan al mejor postor (Rosenberg, 2016, 1).

Es un hecho incontestable que las redes sociales individualizan, empaquetan y sectorializan a sus usuarios. La tecnología, basada en algoritmos, conocida como machine learning, posibilita esta práctica. Ello permite la monetización del tratamiento cualitativo de estos datos, el nuevo petróleo.

En su interacción con las redes sociales, un usuario se ve impelido a dar su consentimiento (de sentire cum) en "licencias de usuario final", de las que desconoce en gran medida su concreto contenido, propósito y alcance.

La multicanalidad, que cristaliza en el uso de formatos multipantalla (por ejemplo, tablets o smartphones) acrecienta el riesgo de esta indefensión, pues a menudo en las pantallas de escasas pulgadas apenas se visualiza una pequeña casilla.

Pero, en un gesto intuitivo, en un clic, el usuario ha dado ya su consentimiento $\mathrm{y}$, presumiblemente, ha cedido muchos derechos que, aun desconociendo, le corresponden.

En Europa, desde principios de 2018, ya se están produciendo las subastas de licitaciones de la red móvil 5G, que espoleará el uso de gadgets tecnológicos y, con ello, la contratación electrónica y, por supuesto, el uso de las redes sociales, la forma de comunicación por excelencia. ¿Pueden revertirse derechos que se han cedido? Si es así, ¿Cuáles? ¿Tendrían carácter retroactivo?

La reciente Sentencia del Tribunal de Gran Instancia de París da la razón a un 
grupo de consumidores de la red social Twitter. Se analizan a continuación los aspectos principales de la misma.

\section{Breve exégesis del caso}

Una asociación de consumidores francesa (en concreto, la Union Fédérale des Consommateurs-UFC) interpone, en nombre y representación de los usuarios/consumidores de la red social Twitter, compañía estadounidense radicada en el Estado de California, una demanda colectiva.

En instancia, todas las pretensiones de la precitada Asociación son desestimadas. La UFC decide apelar las sucesivas decisiones y la demanda sigue su cursus, hasta llegar al Tribunal de Gran Instancia de París.

Presentada la demanda, el Órgano jurisdiccional debe dirimir cuestiones de naturaleza híbrida (procesales y sustantivas).

Entre las primeras, lo primero que debe resolver es la cuestión de la legitimación, cualidad que sirve de base para instar la acción a través de la demanda.

En este sentido, se discute si la Asociación francesa tiene legitimación para actuar en nombre de los usuarios.

En concreto, la elegibilidad de UFC para actuar en nombre de los usuarios de Twitter se fundamentará en el artículo L 621 del Código de Derecho del Consumidor francés (Code du Droit de la Consommation), sobre la base del cual los usuarios de Twitter son considerados consumidores.

La consideración de los usuarios de Twitter como consumidores será disputada por la precitada red social, al argumentar que su servicio es "gratuito", lo que hace imposible que sus usuarios sean "consumidores” (en el sentido de la Ley del Consumidor).

Abundando en lo anterior, la demandada sostiene que es un servicio de microblogging, que presta servicios que consisten en poner a disposición entre sus usuarios mensajes breves (denominados “tuits” y fotografías) gratis, no una red social en sentido estricto. 
De este modo, paraTwitter, una persona sólo puede adquirir la condición de 'consumidor' si paga los productos y servicios contratados.

A propósito de tal condición, para la doctrina mayoritaria el consumidor se limita a participar en la última fase del proceso, sin integrar ningún bien o servicio en actividad productiva alguna, mientras que el empresario, a diferencia del consumidor, adquiere un bien pensando en su valor de cambio (Lasarte: 2014, 50).

Más aún. Tal adquisición responde a un propósito de incorporarlo, generalmente transformado, a su proceso de producción o distribución, a fin de recuperar lo invertido más tarde y multiplicarlo o, en todo caso, incrementarlo; es decir, para obtener nuevos valores de cambio(1).

El Tribunal de París rechaza el planteamiento de la red social al afirmar que, si bien los usuarios de Twitter no "pagan" por el servicio, el uso de su plataforma no es gratuito ${ }^{(2)}$.

En este sentido, la demandada enfatiza que los usuarios otorgan su consentimiento para que su información personal y otros datos sean utilizados por Twitter (y corolario de socios comerciales).

A propósito del consentimiento, recuerda la doctrina consolidada que ciertamente éste consiste en estar de acuerdo o conformes las partes, pero esta conformidad ha de producir la determinación, por lo menos, de todos aquellos extremos del contrato que no estén ya determinados por el Ordenamiento (Albaladejo, 2013, 373).

Es decir, debemos dar por supuesto que el consentimiento debe referirse a los demás elementos objetivos del contrato, como es el objeto que sea materia o causa de él o la causa de las obligaciones que se establezcan (Diez-Picazo, 2007, 174), lo que resulta determinante para la cognoscibilidad jurídica (Díaz-Alabar; 2010, $215)^{(3)}$.

Como contrapartida a la cesión de su consentimiento para que sus datos sean empleados por la red social y su tejido de copartícipes, los usuarios pueden hacer uso de los servicios de Twitter.

Sin embargo, puesto que la red social tiene un claro ánimo de lucro y, por lo 
tanto, tal uso no resulta gratuito, la Ley de Protección del Consumidor se aplica a sus términos y condiciones.

Resuelta la cuestión procesal, el Tribunal entra a decidir sobre el fondo del asunto.

La principal pretensión de la Asociación es que se declare nulo -por abusivo- el clausulado del contrato llamado "Términos \& Condiciones" que vincula al usuario/consumidor con la red social y que se condene a la demandada a resarcir a la parte demandante un millón de euros, en concepto de daños morales.

Recuerda la doctrina mayoritaria francesa que la nulidad sanctionne un dêfautdans la formation du contrat, le quelêtaitdoncaffecté de cette irrégulatritédèsl'origine (por ejemplo, Voirin y Goubeaux, 2016, 403).

Al respecto, el Órgano jurisdiccional francés resuelve la cuestión invalidando gran parte de las cláusulas del referido contrato y condenando a la parte demandada a pagar 30.000 euros, en concepto de daños morales.

\section{Comentario a la Decisión del Tribunal francés}

\section{Aspectos nucleares de la problemática}

En esencia, la cuestiones que conforma el núcleo de la ratio decidendi de la Sentencia se circunscribe a la declaración de nulidad de (una o varias) cláusulas contractuales, una vez determinada su carácter abusivo y el alcance de esta consecuencia.

A continuación, se analiza la fundamentación jurídica de la Sentencia desde una doble perspectiva -procesal y sustantiva.

\section{Aspectos procesales}

Dos son las cuestiones de índole procesal que se discuten en el litigio. La primera, la legitimación de la condición de consumidores. La segunda, el carácter o no abusivo de las cláusulas del contrato “Términos \& Condiciones” (encajamos aquí esta cuestión dado que permanece al albur de un control de oficio por parte del órgano jurisdiccional y, sin su declaración, el resto de las pretensiones del pleito no 
prosperarían).

Una constante de nuestros días es la reivindicación de la unidad de actuación, tanto de los "consumidores" como de otros colectivos (Rosillo, 2010, 497). Aisladamente, en palabras de algún autor, aquéllos no son sino seres "desarmados" (Stiglitz, 2001, 1274), algo que se confirma en el desarrollo de las relaciones jurídicas en la sociedad actual.

Respecto de la primera cuestión, la Ley francesa otorga legitimación para poder ser parte de procesos civiles a los grupos de usuarios o consumidores afectados por un hecho dañoso cuando los individuos que lo compongan estén determinados o sean fácilmente determinables; ello sin perjuicio -como recuerda Rosillo- de que pueda litigarse de forma conjunta ab initio formando un litisconsorcio activo voluntario ${ }^{(4)}$.

Por lo tanto, estamos ante una lista cerrada, tasada. Doctrinalmente, se ha confirmado lo inconveniente de optar por tal lista cerrada (por todos, Vicent, 2000, 6153).

Sin embargo, el Alto Tribunal otorga indubitadamente la legitimación a la Asociación sobre la base legal del artículo 4 de la Directiva 2009/22/CE, del Parlamento Europeo y del Consejo, de 23 de abril de 2009, relativa a acciones de cesación en materia de protección de intereses de consumidores.

Por lo tanto, salva el inconveniente de la lista cerrada del derecho nacional y aplica la legislación europea. No debe olvidarse, al fin y a la postre, que estamos ante un sistema multinivel. Al respecto, como recuerda parte de la doctrina, el TJUE se ha pronunciado sobre la inaplicación de las normas de derecho interno para permitir la efectividad de la norma comunitaria cuando ésta tuviera carácter esencial para el funcionamiento de la UE (por ejemplo, Pérez, 2018, 44).

En concreto, conforme a la normativa comunitaria europea, están legitimadas para ser parte de procesos civiles, en general, y para el ejercicio de las acciones de cesación en defensa de los consumidores colectivos y de los intereses difusos de los consumidores y usuarios -que a l'avantaged' être plus rapidequ' uneaction en réparation et plus souplequ' uneaction en référé- (Dusollier, Francquen, 2015, 132)- las entidades habilitadas ${ }^{(5)}$. 
¿Y de qué entidades habilitadas hablamos? De forma periódica, la Comisión Europea debe publicar en el Diario Oficial de la Unión Europea un listado actualizado de entidades habilitadas, que se conforma con la información suministrada por las autoridades de los Estados Miembros (De la Oliva, 2012, 408).

Salvada la primera cuestión, la segunda será dirimir el carácter o no de abusividad del clausulado del contrato "Términos \& Licencias".

El sistema de protección dispuesto en la legislación comunitaria( ${ }^{(6)}$ se centra en el establecimiento de unos requisitos legales, sin cuyo cumplimiento las condiciones generales no forman parte del contrato; reglas, por lo tanto, de control de incorporación.

A tal efecto, la Norma fija en primer lugar unos requisitos genéricos que deben cumplir las condiciones generales para entender que han quedado incorporadas al contenido del contrato y después señala en qué casos unas condiciones generales no se incorporan al contrato.

Por lo tanto, se exige en primer término, como regla, la aceptación del adherente, expresada habitualmente mediante la firma del contrato en el que debe hacerse referencia a las condiciones generales incorporadas. En todo caso, es indubitado para la doctrina que es preciso que el adherente tenga oportunidad de conocer de manera completa las condiciones generales al tiempo de celebración del contrato (por todos, Martínez de Aguirre et al., 2014, 429).

Se pide, en segundo término, que las condiciones generales sean transparentes, claras, concretas y sencillas.

En este sentido, el Tribunal decide declarar la abusividad de una gran parte de las cláusulas con base en el artículo 4.2 de la Directiva 93/13/CEE, cuyo tenor literal es el siguiente: "La apreciación del carácter abusivo de las cláusulas no se referirá a la definición del objeto principal del contrato ni a la adecuación entre precio y retribución, por una parte, ni a los servicios o bienes que hayan de proporcionarse en contrapartida, por otra, siempre que dichas cláusulas se redacten de manera clara y comprensible".

Dicho de otro modo, no quedarán incorporadas al contrato las condiciones ilegibles, ambiguas, oscuras e incomprensibles. 
Por lo tanto, la exigencia de redacción clara y comprensible es un presupuesto para su conocimiento y, por lo mismo, para la emisión de un consentimiento válido.

En este sentido, el artículo 5 del citado Instrumento normativo exige que las cláusulas sean redactadas siempre de forma clara y comprensible, sancionando, en caso de duda, la prevalencia de la interpretación más favorable para el consumidor.

Abundando en lo anterior, parte de la doctrina (por ejemplo, Ebers, 2008, 201 y 203, y Carballo: 2013, 30) considera que si, en el ámbito de la Directiva [léase la Norma 93/13/CEE, del Consejo, de 5 de abril de 1993], estas cláusulas son inmunes al reproche de un posible carácter abusivo, tal exclusión de control se condiciona a su redacción “de modo claro y comprensible” (ex artículo 4.2), de forma que la cláusula oscura, si no puede salvar su validez por vía de interpretación favorable al consumidor, podrá ser reputada abusiva.

Y, también, hay que apuntar que de la redacción del precepto se colige la intención del legislador europeo en el alcance de los daños y perjuicios que pudieran derivarse de la declaración de nulidad radical en el caso de suministro de bienes y servicios básicos que, por otra parte, en el marco de la tendencia liberalizadora en que actualmente se encuentran inmersos los Estados miembros de la Unión Europea, estos servicios de carácter básico suelen ofrecerse bajo formas monopolísticas, por lo que el daño que comportaría la expulsión de todas las cláusulas de un contrato si eventualmente se declara una nulidad absoluta o radical sería mayor que el daño en sí mismo provocado.

\section{Aspectos sustantivos}

El aspecto nuclear de la ratio decidendi, desde la perspectiva de derecho sustantivo, gira en torno al problema específico de derechos de autor.

El Tribunal de Gran Instancia declara “nulas” la mayoría de las cláusulas impugnadas por el demandante, incluidas las disposiciones de licencia de derechos de autor del contrato para el user-generatedcontent (contenido generado por el usuario).

Los “Términos \& Condiciones” presentan una disposición según la cual los usuarios de Twitter otorgan licencias de derechos de autor a todo el "contenido" que cargan en la plataforma (por ejemplo, texto, fotografías y videos) tanto a la red social como a sus "socios del ecosistema" (es decir, socios externos, terceros, a fin de cuentas). 
La Asociación de consumidores UFC desafía la disposición de la licencia. Su tesis se sustenta en los siguientes aspectos:

1. la fase de apertura da la impresión engañosa de que el acuerdo de licencia es limitado en el alcance de sus subvenciones a Twitter, mientras que de hecho otorga una licencia mundial libre de royalties para cualquier uso y para todo el contenido que se cargue en la plataforma, con el derecho de Twitter de sub-licenciar tales usos a terceros dentro de su "ecosistema" comercial;

2. la cláusula es tan amplia que contraviene al Code de la propriété intellectuelle(Código de Propiedad Intelectual francés), que proporciona un tratamiento más estricto de lo que se puede autorizar o asignar que lo que hacen los países de Common Law. Estas reglas se introdujeron para salvaguardar los intereses de los autores los cuales, en este caso, se quedaban alienados sin justificación legal;

3. el Code de la propriété intellectuelle prohíbe la asignación o licencia de obras futuras (nulidad), $\mathrm{y}$

4. la Norma precitada también exige que las transferencias de derechos (asignaciones o licencias) se registren por escrito y sean muy específicas en cuanto a su contenido. Es un requisito legal que cada derecho "transferido" se mencione por separado en el contrato y que el alcance, el propósito, el lugar y la duración de la explotación para cada uno de estos derechos estén claramente definidos en el acuerdo para que la disposición pueda ser válida;

Cabe subrayar en este punto que la provisión general de licencia de Twitter no cumplía con ninguno de los requisitos señalados.

Respecto a la primera consideración, es preciso señalar que la Asociación de consumidores sostiene que la primera cláusula de la licencia de Twitter advierte al usuario de lo siguiente: "Youre tain your rights to any Content you submit, post or display o north rough the Services. What's your sisyours - you own your Content (and your incorporated audio, photos and videos are considered partof the Content)” [sic], lo que se contradice con lo que la Plataforma hace de facto.

Abundando en lo anterior, nos encontramos ante una situación en la que el cedente -el usuario de la red social en este caso- otorga todos los derechos que pudieran corresponderle en exclusiva. Se trata de una situación de hecho que se 
contrapone a la condición de titular originario o de iure.

Recuerda la doctrina consolidada que el cesionario de los derechos de autor, en cuanto goza de un derecho de explotación patrimonial, resulta el sujeto pasivo de los atentados que afecten a dicho interés patrimonial (por todos, De Nova Labián, 2010, 137).

En relación a las cuestiones segunda y tercera, el Alto Tribunal señala que: "La cláusula que otorga al proveedor del servicio el derecho a utilizar, de forma gratuita, cualquier contenido generado por el usuario, incluido el contenido que puede estar protegido por derechos de autor, sin especificar con suficiente detalle el contenido en cuestión, la naturaleza de los derechos transferidos y la naturaleza de los usos comerciales acordados infringe las disposiciones de los artículos L 131-1, L 131-2 y L 131-3 del Código de Propiedad Intelectual" (FJ3º).

En efecto, el apartado primero del artículo 131 del CPI francés contiene unas formalidades estrictas. En concreto, prescribe la exigencia de que las condiciones sean escritas y que contengan los siguientes extremos [sic]: "le détail de chacun des droits cédes, le détail des modesd'explotation des droits cédé squant à leurétendue et leurdestination, le territoire pour le quel les droits son décès, la durée, la remunération".

De este modo, tras recordar que el CPI prohíbe la cesión global de obras futuras (apdo. tercero del mismo precepto), advierte la doctrina que ilconvientdoncd'êtreparticulièrementlorsqu'ils'agitd'uneoeuvrecréée par plusiersauters: leurscontractsdevontêtre en toutpointidentiquesdansleurpérimètre de la cession (por todos, Riahi, 2013, 25).

La última cuestión es, sin duda, la más relevante y abre una nueva era en la concreta cuestión de los derechos inherentes a los contenidos generados por los consumidores/usuarios.

Un último apunte sobre la cuestión de los derechos morales. Parte de la doctrina clásica ha justificado el derecho moral como garante del interés público (por ejemplo, Recht, 1949, 3). Esta opinión se sitúa en línea con un importante sector de la doctrina italiana, que ha puesto de relieve el papel que cumple el interés público en la tutela del derecho de autor (en este sentido, De Sanctis: 1959, 400). Evidentemente, consideramos que la sanción económica impuesta a Twitter es discreta y se 
aleja considerablemente de otras soluciones judiciales que suelen aplicar la "regla del tercio". Sin embargo, no debe olvidarse que los daños punitivos son propios del derecho anglosajón.

Probablemente, el órgano jurisdiccional habrá tenido en cuenta para el cálculo del quantum indemnizatorio las previsiones contenidas en el artículo L 131-4 del Code, que contempla un elenco de supuestos generales y que contempla, a modo de cláusula de cierre, la previsión de incluir el tanto alzado en supuestos que no son identificables.

Para parte de la doctrina considera, de hecho, que el art. L 131-4-apartado $2^{\circ}$ es aplicable a todos aquellos casos en los que el cesionario no puede determinar los ingresos brutos de la explotación de la obra, teniendo en cuenta que son precisamente esos mismos ingresos los que sirven de base para el cálculo de la remuneración proporcional (Cabedo, 2011, 250).

A ello debe añadirse que, habida cuenta que, aunque la vía judicial civil francesa sea expeditiva, la cuestión probatoria, que recae sobre el demandante, continúa siendo harto complicada.

En sede de propiedad intelectual, la responsabilidad no es objetiva y se precisa un elemento de culpa (Mallo: 2018, 362), por lo que consideramos que la Sentencia es ajustada a derecho, pues lo importante es que actúe de palanca disuasoria profuturo; al respecto, there is and there will be ample space for the European Cour to Justice to shape the constitutionalisation of European prívate law and hopefully to provade 'general principles` which would start to connect the fragmented bits and pieces of EU law (Leczykiewicz y Weatherill, 2018, 41).

\section{Conclusiones}

Hasta ahora, los propietarios de los códigos del software y, en general, de todo el contenido generado por los usuarios eran los dueños de las plataformas.

La Sentencia del Gran Tribunal de París da un vuelco a esta situación por cuanto, al menos en Francia, Twitter deberá adecuar el contenido de sus acuerdos a la legislación francesa, para dar cumplimiento de la citada Resolución, que declara la nulas la mayor parte de las cláusulas que venía empleando la red social. 
Las dos cuestiones más relevantes que se desprenden de esta Resolución son la estimación de la legitimación para reclamar en calidad de consumidor y el reconocimiento de los derechos de propiedad intelectual.

Respecto de la primera cuestión, consideramos acertada la Decisión de la Sala, que aplica el artículo Directiva 2009/22/CE, del Parlamento Europeo y del Consejo, de 23 de abril de 2009, relativa a acciones de cesación en materia de protección de intereses de consumidores.

La legislación nacional y comunitaria también cuenta con resortes suficientes para accionar una demanda colectiva. Podría haberse sustentado en la legitimación que ostentan entidades como las cámaras de comercio, figuras ampliamente reconocidas en muchos ordenamientos jurídicos nacionales.

Contribuye a la afirmación anterior el reconocimiento constitucional de la protección de los intereses económicos de los consumidores, ampliamente reconocido en las cartas magnas europeas.

En cualquier caso, se evidencia la influencia anglosajona de las classactions, lo que pone de relieve que el Derecho continental está vivo y es cada vez más poroso y proclive a su incorporación.

A partir de esta importante Decisión, se abre una vía para que los usuarios/consumidores reclamen sus derechos en materia de propiedad intelectual. En concreto, por derechos inherentes al contenido que puedan generar en el marco de estas plataformas.

Una Resolución, como puede comprobarse, muy relevante y que traerá cola, habida cuenta que en el ecosistema digital los usuarios que generan sus propios contenidos tienen cada vez mayor presencia. 


\section{Referencias}

Albadalejo, M. (2013). Derecho de obligaciones. Madrid: Edisofer.

Bergé, J. S. (2015). La protection internationale et européenne du droit de la proprieté intellectuelle. Bruselas: Larcier.

Cabedo Serna, L. (2011). El derecho de remuneración del autor. Madrid: Dykinson.

Carballo Fidalgo, M. (2013). La protección del consumidor frente a las cláusulas no negociadas individualmente. Disciplina legal y tratamiento jurisprudencial de las cláusulas abusivas. Barcelona: Bosch.

Díez-Picazo, L. (2007). Fundamentos del derecho civil patrimonial I. Pamplona: Aranzadi. Thomson Civitas.

Dusollier, S. y Francquen, A. (2015). Manuel de droits intellectuels. Bruselas: Anthemis.

Ebers, M. (2009). “Un fair Contract Terms Directive 93/13” En H. Schulte-Nölke, C. Twigg, Flesner, M. Ebers (eds.), EC Consumer Law Compendium. The Consumer Acquis and its Transposition in the Member States. Munich: Sellier.

Lasarte Álvarez, C. (2014). Manual sobre protección de consumidores y usuarios ( $6^{\circ}$ ed.). Madrid: Dykinson.

Leczykiewicz, D. y Weatherill, S. (2018). The images of the Consumer in the EU Law. Oxford: Hart.

Mallo Montoto, D. (2018) La difusión en Internet de contenidos sujetos al derecho de autor. Madrid: Reus. (Col. de Propiedad Intelectual).

Martínez de Aguirre, C. et al. (2014). Derecho de obligaciones (4a ed.). Madrid: Colex.

De Nova Labián, A. (2010). Delitos contra la propiedad intelectual en el ámbito de internet. Madrid: Dykinson. 
De Sanctis (1959). Enciclopedia del Diritto. Roma: Giuffre Editore.

Pérez Daudí, V. (2018) La protección procesal del consumidor y el orden público comunitario. Barcelona: Atelier.

Recht (1949). Reflexions sur la protection du droit d'auteur (Vol. 2). París: BDA.

Riahi, K. (2013). Les contrats de la production. París: Dixit.

Romero Robredo, A. (2017). Privacidad e intimidad en las redes sociales. Madrid: Re-Unir.

Rosenberg, M. (2016). The Price of Privacy: How access to digital privacy is slowly be coming divided by class. UCLA: Journal of Law \& Technology.

Rosillo Fairén, A. (2010). La configuración del contrato de adhesión con consumidores. Madrid: La Ley.

Stiglitz, G. (1991). El Derecho contractual y la protección jurídica del consumidor en América Latina. Madrid: Instituto Nacional de Estudios Jurídicos

Vicent Chuliá, F. (2000). Las acciones colectivas de condiciones generales y su impacto en los sectores de contratación especial. Revista General de Derecho, 56(663).

Voirin, P. y Goubeaux, G. (2016). Droit civil (36ª éd.). París: Lex tenso éditions.

\section{Notas}

${ }^{1}$ Ibidem.

2 “Aux termes des articles 1105 et 1106 devenul'article 1107 du code civil, le contratestdit à titreonéreux, lors que chacune des partiesreçoit de l'autre un avantage en contrepartie de celuiqu'elle procure, ilestdit à titregratuit, lors quel'une des parties procure à l'autre un avantage, sansrecevoir de contrepartie. Ilressort des documents relatif saux Conditions Générales d'utilisation et de la Politique de confidentialité de Twitter, produits audébat, qu'encontrepartie de l'accès et de l'utilisation des Services Twitter, l'utilisateuraccepte que 
Twitter, sesfournisseurs et ses partenaires puissent placer des publicitésciblées en fonction des requêteseffectuéesaumoyen des services (clausen ${ }^{\circ} 1.5$ des Conditionsd'utilisation du é( juin 2012, 8 septembre 2014, 18 mai 2015 et 27 janvier 2016, devenu la clausen ${ }^{\circ} 4.3 .1$ du 30 setembre 2016) ; que les services de Twitter sontfinancés par la publicité, la société Twitter étant susceptible d'utiliser les informationsdécritesdanssaPolitique de confidentialitépour que les publicités soient plus pertinentes, pour en mesurerl'efficacité et pouraider à reconnaître les appareils de l'utilisateurafin de lui diffuser des annonces sur et hors Twitter (clausen ${ }^{\circ} 16$ de la Politique de confidentialité du 30 septembre 2016) ; que Twitter collecte, utilise les informations que l'utilisateuradresselors de son utilisation de sesServices [...]”. (FJ2 $\left.{ }^{\circ} \mathrm{A}\right)$.

${ }^{3}$ Díaz-Alabart, S. en Rosillo, A. (2010) La configuración del contrato de adhesión con consumidores. Madrid: La Ley, pág. 25.

${ }^{4}$ Op. Cit. Pág. 574.

${ }^{5}$ En concreto, el Tribunal confiere la legitimación en aplicación de lo siguiente [sic]: "L'article L. 421-6 du code de la consommation, devenul'article L. 621-7 du code de la consommation, prévoit que les associationsmentionnées à l'article L. 421-1 devenul'article L. 621.1 du code de la consommation et les organismesjustifiant de leurinscription sur la liste publiéeauJournalofficiel de l'Unioneuropéenne en application de l'article 4 de la directive 2009/22/ CE du Parlementeuropéen et du Conseil du 23 avril 2009 modifiée, relativeauxactions en cessation en matière de protection des intérêts des consommateurs, peuventagirdevant la juridictioncivilepour faire cesserouinterdiretoutagissementilliciteauregard des dispositionstransposant les directivesmentionnées à l'article 1er de la directiveprécitée" (FJ1º).

${ }^{6}$ En concreto, la Directiva 93/13/CEE, del Consejo, de 5 de abril de 1993, sobre las cláusulas abusivas en los contratos celebrados con consumidores y la Directiva 2011/83/UE del Parlamento europeo y del Consejo, de 25 de octubre de 2011, sobre los derechos de los consumidores, por la que se modifican la directiva 93/13/CEE del Consejo y la Directiva 1999/44/CE, del Parlamento europeo y del Consejo y se derogan la Directiva 85/577/CEE, del Consejo y la Directiva 97/7/CE, del Parlamento europeo y del Consejo. 